\title{
Preplacement low-back screening for high-risk areas
}

\author{
KAREN M STEELE, DO
}

VC HOEFNER, JR., DO, FAAO

\begin{abstract}
A minimum level of health is required before a worker is placed in an area defined as high risk for low-back injury. Adequate employee fitness may be defined by three criteria: weight, to within the eightieth percentile; passage of eight strength and flexibility tests; and normal results on neurologic examination. The results are reproducible and require little subjective interpretation by the examiner. Ability to meet these minimum standards for heavy laborers should help to decrease the incidence of low-back pain and injury.
\end{abstract}

The importance of screening persons at risk for low-back injuries can be appreciated when we realize that 70 to $85 \%$ of adults in the United States will experience at least one episode of low-back pain. ${ }^{1-3}$ It is estimated that $20 \%$ of back injuries each year occur at work. ${ }^{4}$ The total cost of caring for these occupational injuries is estimated at 30 billion dollars each year in the U.S. alone. ${ }^{4,5}$ Knowing that the average age for the first incidence of low-back pain is from 30 to 35 years, ${ }^{5}$ and that the likelihood of a disabled employee returning to the work force after 6 months of disability is only 50 percent, ${ }^{5}$ this represents a staggering finanical loss to industry.

Most employers are concerned with reducing the incidence of on-the-job injury, lost work time, and disability claims. Large industrial firms have company physicians who screen prospective employees to determine ability to safely perform the job for which they are applying. However, two thirds of the companies in the United States are small, and the family physician frequently will be asked by either a patient or a company to evaluate an employee before placement.

The physician who performs preplacement lowback screening must guard against discrimination. The chance to apply for a specific job should never be prohibited because the patient is handicapped, female, or small in stature or has any other physical attribute without testing that person for the task. Many persons denied employment have sought legal counsel on the basis of discrimination. Large companies spend huge sums of money defining the specific physical requirements for safe performance of a given task in the plant. The requirements are based on a statistical analysis of the predictive value of passage of a certain testing situation as related to the ability to perform that task without injury. This very expensive and time-consuming procedure is not feasible in the small plant or private physicians' office.

The question then becomes how the private practitioner should proceed when asked to screen an employee. Obviously, the physician is being drawn into the complex and controversial field of industrial medicine.

From the osteopathic physician's point of view, a good general overall level of health and biomechanical stability of the low back and pelvis must be an important prerequisite to safe performance of a task. Most back rehabilitation programs address these two areas intensively after an injury.

Following is a proposal for preplacement back screening that places significant emphasis on overall fitness of the applicant-mental as well as physical. The applicant is screened for ability to meet minimum standards. Certain deficiencies must be conditioned, improved, or corrected; then the applicant is ready for specific task testing if needed. No special equipment or space is required other than what is already available in the practitioner's office. By improving the known factors contributing to industrial low-back injury, the physician should be able to decrease such injury both on and off the job.

\section{Screening procedure}

The routine history should be elicited first and should include assessment of usage of tobacco, which is associated with increased risk of lowback pain, ${ }^{4,5}$ alcohol, and any prescription, nonprescription, or recreational drugs. The patient's usual way of dealing with stressors and participation in any exercise program should be noted. A detailed history of previous episodes of low-back pain should be taken including the cause (traumatic or nontraumatic), length of recovery, and 


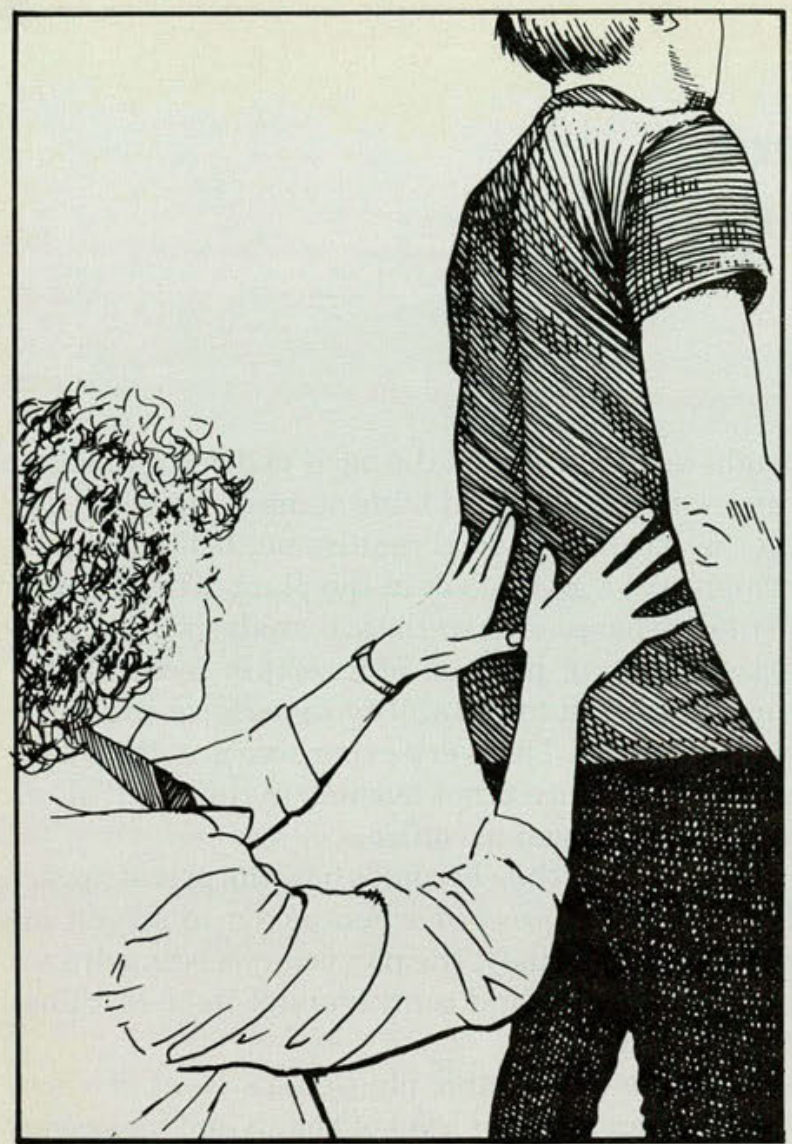

Fig 1. Screen for posture and attendant lumbar lordosis.

treatment modalities used..$^{5}$

The physical examination, with recording of height and weight, follows. Any deviations from the norm should be noted, especially weight above the eightieth percentile for age. At a lumbosacral angle of 30 degrees, the shear stress between the fifth lumbar vertebra and the sacral base is $50 \%$ of the superimposed weight. Increasing the angle to 50 degrees increases the shear stress to $75 \%$ of superimposed weight. ${ }^{3}$ Weight gain usually causes postural faults, such as anterior weight bearing, as well as increases the shear stress between L-5 and the sacrum. Spondylosis, spondylolysis, spondylolisthesis, and facet degeneration are common complications of the increased shear force between the lumbar spine and sacrum. ${ }^{3}$

Posture should be examined from the posterior and lateral aspects, with special note made of scoliosis, adaptation to short leg, and an important increased lumbar lordosis (Fig 1). The latter is the altered finding that suggests the possibility of postural faults and poor body which frequently contribute to low-back pain. ${ }^{6}$ Posture is also a somatic reflection of the emotions. ${ }^{2}$ Depression, for example, may be shown by "droopy" posture that both

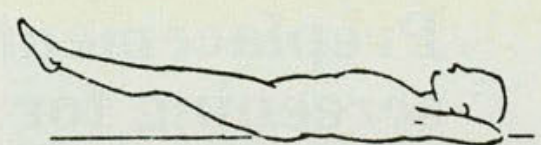

Fig 2. Test for assessing strength of hip flexors (from Kraus ${ }^{7}$ ). (Figs 2 to 7 Reprinted with permission. Copyright 1965 by Simon and Schuster, New York.)

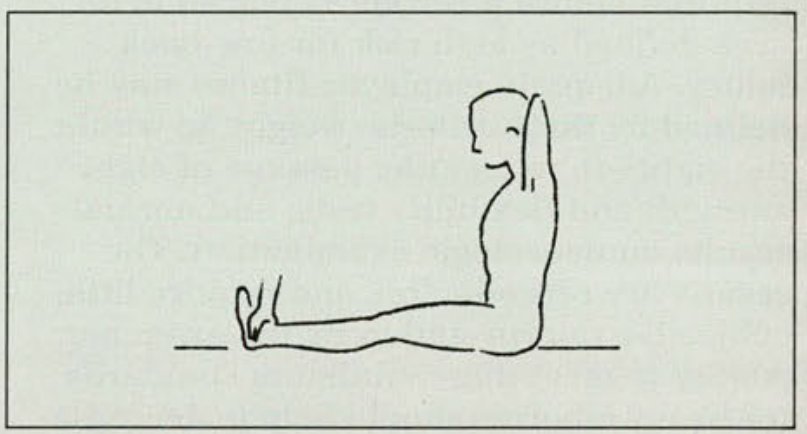

Fig 3. Test for assessing the strength of hip flexors and abdominal muscles (from $\mathrm{Kraus}^{7}$ ).

expresses and produces fatigue. Important cues into a person's mental health can be obtained from the postural evaluation.

The screening of the minimum level of strength and flexibility required to move the body against gravity has been described by Kraus. ${ }^{7} \mathrm{He}$ indicated six simple maneuvers (Kraus-Weber test) for assessing the strength of hip flexors, abdominal muscles, and upper and lower back muscles, and flexibility of low-back and thigh muscles.

These tests are performed as follows: (1) Hip flexor strength is determined by having the patient hold the legs off the floor with knees straight, 10 seconds while supine (Fig 2). (2) The strength of hip flexors and abdominal muscles combined is determined by having the patient do one sit-up with ankles stabilized and knees straight (Fig 3). (3) The strength of abdominal muscles is tested by having the patient do one sit-up with ankles stabilized and knees bent (Fig 4). (4) Upper back strength is assesed by having the patient hold the trunk off the floor for ten seconds while prone with ankles and low-back stabilized (Fig 5). (5) The strength of lower back muscles is evaluated by having the patient, while prone, hold the legs off the floor for ten seconds (Fig 6). (6) Low-back/posterior thigh flexibility is tested by having the patient do one straight-leg toe touch (Fig 7).

Kraus $^{7}$ stressed that the ability to pass these 


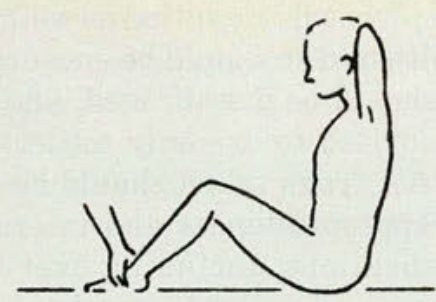

Fig 4. Maneuver for determining strength of abdominal muscles (from $\mathrm{Kraus}^{7}$ ).

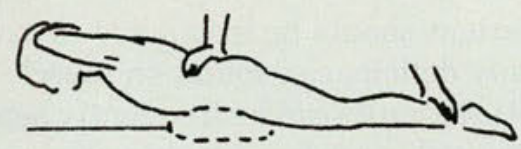

Fig 5. Upper back strength test (from Kraus ${ }^{7}$ ).

screening tests does not imply conditioning sufficient to engage in heavy labor or strenuous sports. However, a surprising number of workers are unable to perform even these maneuvers. Two additional screening tests are suggested to the reader: hip flexor (psoas) flexibility and quadriceps strength testing. The flexibility of the hip flexors is tested by having the patient prone on the table and passively extending the hip. Anything less than $30^{\circ 8}$ is indicative of hip flexor shortening, which can be associated with postural faults. ${ }^{9}$ The quadriceps strength is tested by having the patient lean against the wall with hips and knees flexed $60^{\circ}$ to $90^{\circ}$ (Fig 8) and holding this position for as long as possible. Ideally, the patient should be able to hold this position for 3 minutes. ${ }^{5}$ Quadriceps strength is necessary for many safe lifting techniques utilizing the legs rather than the low back. If minimal strength and flexibility requirements are not being met, how can one expect workers to engage safely in heavy labor?

A screening neurologic examination should be done to include deep tendon reflexes, sensory test of the L2 to S1 dermatomes, and strength testing of the lower extremities. Spinal myotomes can be used to screen L2 to S2 in these maneuvers. ${ }^{10}$ With the patient standing, the ability to contract the hip flexors (L2 to L3) and hip extensors (L4 to L5) against counterforce assessed with the patient

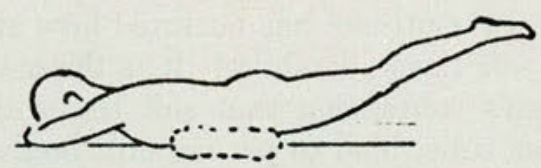

Fig 6. Maneuver for testing strength of lower back muscles (from Kraus $^{7}$ ).

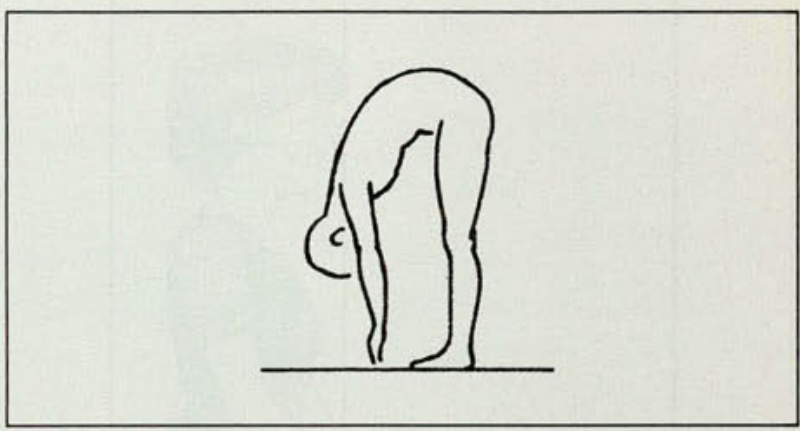

Fig 7. Maneuver for assessing low-back/posterior thigh flexibilty (from Kraus ${ }^{7}$ ).

seated, and myotomes $\mathrm{S} 1$ to $\mathrm{S} 2$ are screened by having the patient plantar flex against your resistance. Deficiencies, of course, require further investigation.

Somatic dysfunction of the lumbar spine and pelvis must be screened and treated in order to improve the biomechanical functioning of the worker. Nonphysiological conditioning such as unilateral sacral flexion ${ }^{11}$ (inferior sacral shear) ${ }^{12}$ are significant in low-back pain and should be treated if found.

Radiologic evaluation should be a part of screening only if the physical examination or history suggests the need for a special diagnostic procedure. The standing postural study with lumbar oblique views, as described by Willman, ${ }^{13}$ is suggested. Attention should be directed to calculated values that reflect altered lordosis (weight-bearing line, lumbosacral angle, and pelvic index). The presence of scoliosis, spondylolysis, spondylolisthesis, short leg, congenital anomalies, and bone or metabolic diseases evident in the radiograph should be noted.

\section{Conditioning}

Failure to meet any of these minimum standards of strength and flexibility should alert the physician to the employee's risk for low-back pain and injury. Following are recommendations for conditioning or improving the applicants' ability to meet 
these minimum health standards.

High levels of emotional or physical stress should be reduced. Cailliet ${ }^{2}$ has outlined how stress can lead to soft tissue disability. It is the osteopathic physician's contention that soft tissue disability can, over time, lead to permanent, nonreversible

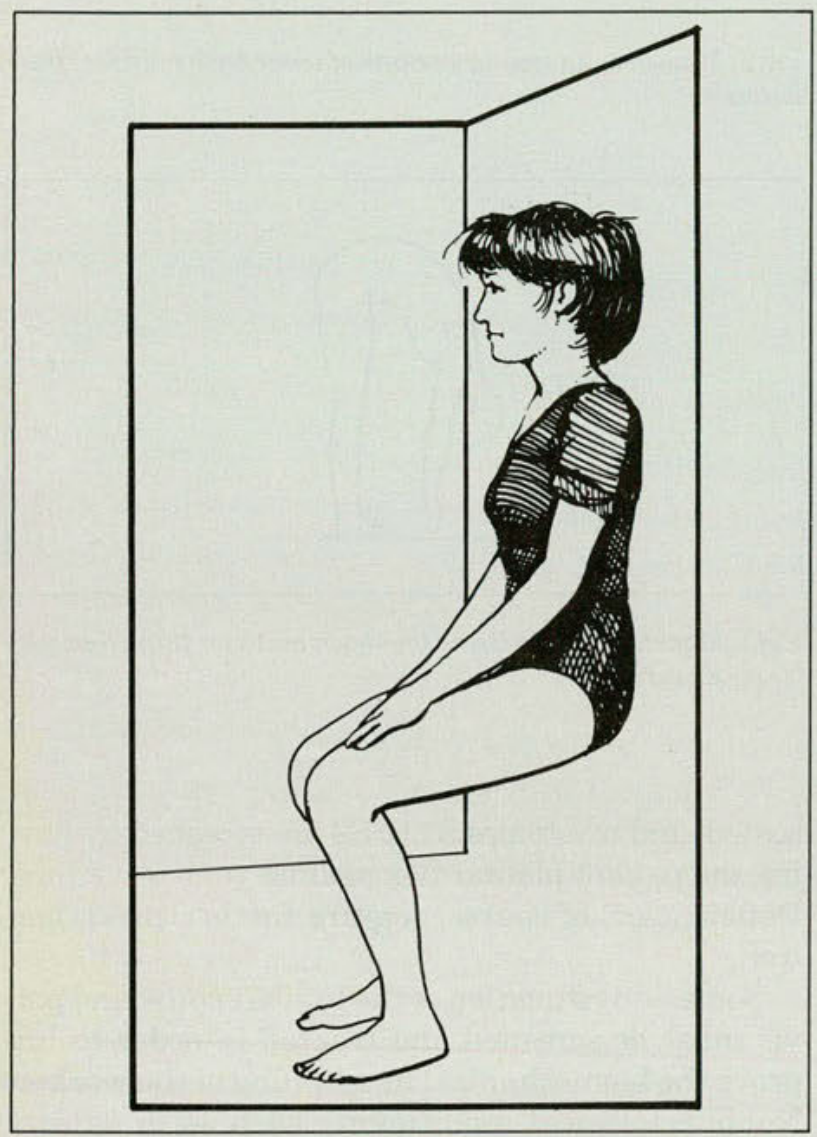

Fig 8. Wall slide. disability. A regular exercise program can provide mental and physical relaxation as well as improved overall health and so should be encouraged. Usage of tobacco should be discouraged, and the patient should be advised to use only moderate amounts of alcohol. All drugs taken should be reviewed to determine appropriateness and interactions. Use of pain medications, including over-the-counter preparations on a regular basis, should be discouraged. All recreational drug use should be discontinued.

Weight loss is indicated if weight is above the eightieth percentile for age. The significance of obesity in predisposing to postural derangements should be stressed, and the overweight patient should have an eating re-education program prescribed.

The patient should be instructed in exercises to correct any deficiencies found on muscle strength test. Postural faults can be frequently benefited by the "pelvic coil" or pelvic tilt exercise in which the patient straightens the back, holds the stomach in, contracts the gluteii, and brings the pubic ramus superiorly. 2,5 This exercise decreases lumbar lordosis while strengthening postural muscles needed for pain-free motion. The abdominal muscles can be conditioned by the "crunch" or partial sit-up with knees flexed (Fig 9). This position is held for 1 minute. ${ }^{5}$ The quadriceps muscle group can be strengthened by having the patient assume the "wall slide" (Fig 8) position daily, and encouraging 3 minutes or more of sustained effort.

Flexibility of the psoas muscle can be increased by having the patient lean against a door edge, reaching behind, and grasping the door knob for

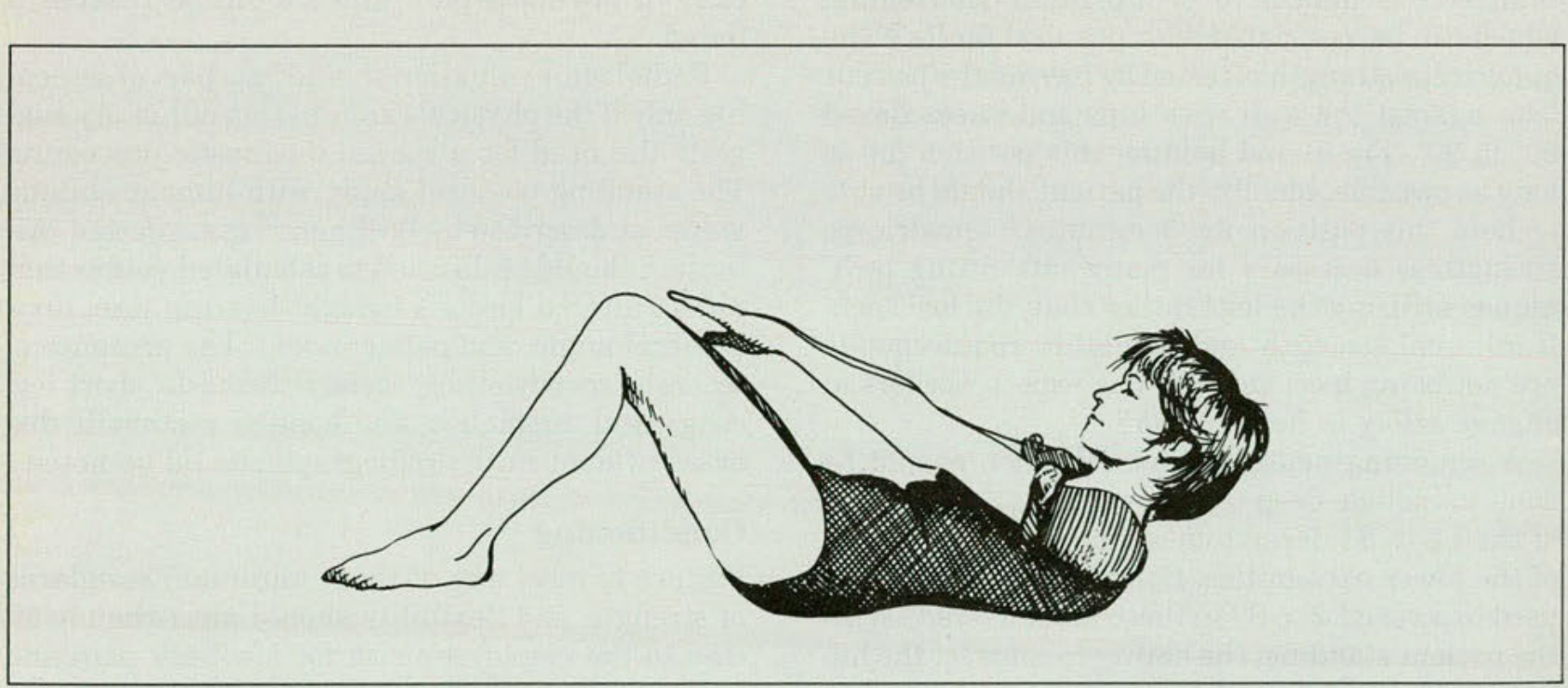

Fig 9. Partial sit-up. 


\section{non-insulin-dependent}

\section{Diabetes and fasting glucose}

Fasting hyperglycemia is largely due to hepatic overproduction of glucose. MicRONASE has been shown to decrease elevated basal rates of hepatic glucose production, and this may account for its ability to reduce fasting hyperglycemia.

MiCRONASE improves 24-hour control of both postprandial and fasting blood glucose levels. MiCRONASE usually provides 24-hour control of blood glucose levels with a once-a-day dosage.

All sulfonylureas, including MicronAsE, can cause severe hypoglycemia. Proper patient selection, dosage, and instructions are important.

No other oral antidiabetic agent fits the realities of life better than

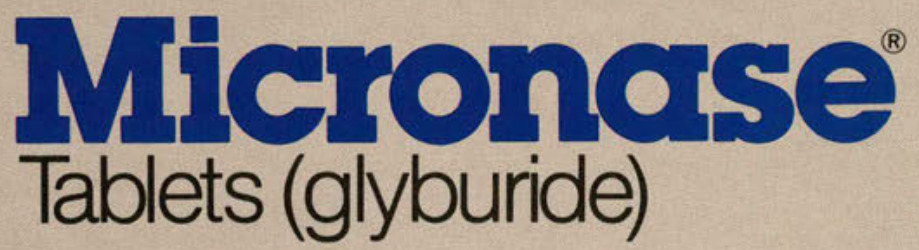

Usual starting dosage $-2.5 \mathrm{mg}$ to $5 \mathrm{mg}$ once a day

When diet alone fails...MicronASE

Please see adjacent page for brief summary of prescribing information.

\section{Upjohn}



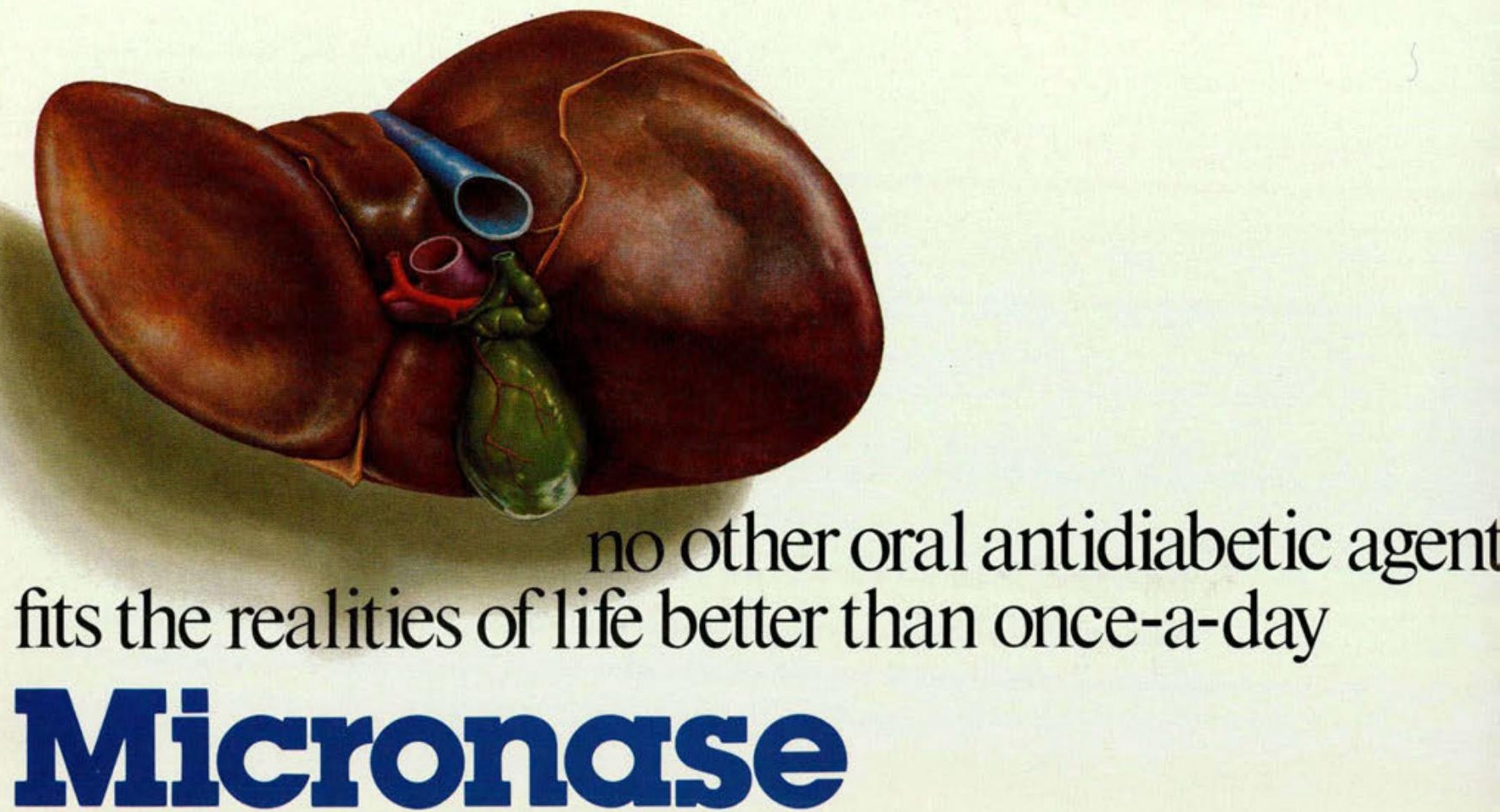

Some patients, particularly those receiving more than $10 \mathrm{mg}$ daily, may

have a more satisfactory response with twice-a-day dosage.

\section{Micronase Tablets (glyburide)}

CONTRAINDICATIONS: MICRONASE Tablets are contraindicated in patients with: 1. Known hypersensitivity or allergy to the drug. 2. Diabetic ketoacidosis, with or without coma. This condition should be treated with insulin. 3. Type I diabetes mellitus, as sole therapy.

SPECIAL. WARNING ON INCREASED RISK OF CARDIOVASCULAR MORTALITY: The administration of ora hypoglycemic drugs has been reported to be associated with increased cardiovascular mortality as compared to treatment with diet alone or diet plus insulin. This warning is based on the study conducted by the University Group Diabetes Program (UGDP), a long-term prospective clinical trial designed to evaluat the effectiveness of glucose-lowering drugs in preventing or delaying vascular complications in patients with noninsulin-dependent diabetes. The study involved 823 patients who were randomly assigned to one of four treatment groups (Diabetes, 19 [Suppl 2]:747-830, 1970). UGDP reported that patients treated for 5 to 8 years with diet plus a fixed dose of tolbutamide ( 1.5 grams per
day) had a rate of cardiovascular mortality approximately $2 \frac{1}{2}$ times that of patients treated with diet alone. day) had a rate of cardiovascular mortality approximately $2 \frac{1}{2}$ times that of patients treated wittrdiet alone. based on the increase in cardiovascular mortality, thus limiting the opportunity for the study to show an increase in overall mortality. Despite controversy regarding the interpretation of these results, the finding of the UGDP study provide an adequate basis for this warning. The patient should be informed of the potential risks and advantages of MICRONASE and of alternative modes of therapy.

Although only one drug in the sulfonylurea class (tolbutamide) was included in this study, it is prudent from a safety standpoint to consider that this warning may apply to other oral hypoglycemic drugs in this class. in view of their close similarities in mode of action and chemical structure.

PRECAUTIONS: General-Hypoglycemia: All sulfonylureas are capable of producing severe hypoglycemia Proper patient selection and dosage and instructions are important to avoid hypoglycemic episodes. Renal or hepatic insufficiency may increase the risk of serious hypoglycemic reactions. Elderly, debilitated or malnourished patients, and those with adrenal or pituitary insufficiency, are particularly susceptible to the hypoglycemic action of glucose-lowering drugs. Hypoglycemia may be difficult to recognize in the elderly and in people who are taking beta-adrenergic blocking drugs. Hypoglycemia is more likely to occur when caloric intake is deficient, after severe or prolonged exercise, when alcohol is ingested, or when more than one glucose lowering drug is used. Loss of Control of Blood Glucose: In diabetic patients exposed to stress such as fever, trauma, infection or surgery, a loss of control may occur. It may then be necessary to discontinue MICRONASE and administer insulin. Adequate adjustment of dose and adherence to diet should be assessed before classifying a patient as a secondary failure. Information for Patients: Patients should be informed of the potential risks and advantages of MICRONASE and of alternative modes of therapy. They also should be informed about the importance of adherence to dietary instructions, of a regular exercise program, and of regular testing of urine and/or blood glucose. The risks of hypoglycemia, its symptoms and treatment, and conditions that predispose to its development should be explained to patients and responsible family members. Primary and secondary failure should also be explained. Laboratory Tests: Response to MICRONASE Tablets should be monitored by frequent urine glucose tests and periodic blood glucose tests Measurement of glycosylated hemoglobin levels may be helpful in some patients. Drug Interactions: The hypoglycemic action of sulfonylureas may be potentiated by certain drugs including nonsteroidal antiinflammatory agents and other drugs that are highly protein bound, salicylates, sulfonamides, chloramphenicol, probenecid, coumarins, monoamine oxidase inhibitors, and beta adrenergic blocking agents. Certain icol, probenecid, coumarins, monoamine oxidase inhibitors, and beta adrenergic blocking agents. Certain
drugs tend to produce hyperglycemia and may lead to loss of control. These drugs include the thiazides and other diuretics, corticosteroids, phenothiazines, thyroid products, estrogens, oral contraceptives, phenytoin, nicotinic acid, sympathomimetics, calcium channel blocking drugs, and isoniazid. A potential interaction between oral miconazole and oral hypoglycemic agents leading to severe hypoglycemia has been reported Carcinogenesis, Mutagenesis, and Impairment of Fertility: Studies in rats at doses up to $300 \mathrm{mg} / \mathrm{kg} / \mathrm{day}$ for 18 months showed no carcinogenic effects. Glyburide is nonmutagenic when studied in the Salmonells microsome test (Ames test) and in the DNA damage/alkaline elution assay. Pregnancy: Teratogenic Effects: Pregnancy Category B. Reproduction studies in rats and rabbits have revealed no evidence of impaired fertility or harm to the fetus due to glyburide. There are no adequate and well controlled studies in pregnant women. This drug should be used during pregnancy only if clearly needed. Insulin should be used during pregnancy to maintain blood glucose as close to normal as possible. Nonteratogenic Effects: Prolo severe hypoglycemia ( 4 to 10 days) has been reported in neonates born to mothers who were receiv sulfonylurea drug at the time of delivery. MICRONASE should be discontinued at least two weeks befor expected delivery date. Nursing Mothers: Some sulfonylurea drugs are known to be excreted in human Insulin therapy should be considered. Pediatric Use: Safety and effectiveness in children have not Insulin therapy
established

ADVERSE REACTIONS: Hypoglycemia: See Precautions and Overdosage sections. Gastrointestinal tions: Cholestatic jaundice may occur rarely: MICRONASE Tablets should be discontinued if this oc Gastrointestinal disturbances, e.g. nausea, epigastric fullness, and heartburn, are the most con reactions, having occurred in $18 \%$ of treated patients during clinical trials. They tend to be dose-relate may disappear when dosage is reduced. Liver function abnormalities, including isolated transam elevations, have been reported. Dermatologic Reactions: Allergic skin reactions, e.g. pruritus, eryth urticaria, and morbilliform or maculopapular eruptions, occurred in $1.5 \%$ of treated patients during ci trials. These may be transient and may disappear despite continued use of MICRONASE; if skin reac persist, the drug should be discontinued. Porphyria cutanea tarda and photosensitivity reactions have reported with sulfonylureas. Hematologic Reactions: Leukopenia, agranulocytosis, thrombocytop hemolytic anemia, aplastic anemia, and pancytopenia have been reported with sulfonylureas. Meta Reactions: Hepatic porphyria and disulfiram-like reactions have been reported with sulfonylureas: how hepatic porphyria has not been reported with MICRONASE and disulfiram-like reactions have been rep very rarely. Cases of hyponatremia have been reported with glyburide and all other sulfonylureas, most in patients who are on other medications or have medical conditions known to cause hyponatremia or inc release of antidiuretic hormone. (SIADH) secretion has been reported with certain other sulfonylureas it has been suggested that these sulfonylureas may augment the peripheral (antidiuretic) action of ADF /or increase release of ADH.

OVERDOSAGE: Overdosage of sulfonylureas, including MICRONASE Tablets, can produce hypoglycen hypoglycemic coma is diagnosed or suspected, the patient should be given a rapid intravenous inject concentrated ( $50 \%$ ) glucose solution. This should be followed by a continuous infusion of a more dilute glucose solution at a rate which will maintain the blood glucose at a level above $100 \mathrm{mg} / \mathrm{dL}$. Patients st be closely monitored for a minimum of 24 to 48 hours, since hypoglycemia may recur after apparent cl recovery.

Caution: Federal law prohibits dispensing without prescription. Store at controlled room temper $15^{\circ}-30^{\circ} \mathrm{C}\left(59^{\circ}-86^{\circ} \mathrm{F}\right)$. Dispensed in well closed containers with safety closures. Keep container tightly clo For additional product information see your Upjohn representative.

\section{Upjohn}

THE UPJOHN COMPANY, Kalamazoo, MI 49001, USA 
support. The hip is extended as far as possible and held for a count of ten (with five repetitions) at least once daily. Hamstring flexibility can be maintained or increased by having the patient do single leg lifts with the opposite knee bent, holding the leg as high as possible for five seconds (with fiveten repetitions) at least once daily. ${ }^{7}$ Stretches should also be done prior to any lifting,,$^{1,7}$ especially after sitting for one-half hour or longer.

Somatic dysfunction identified should be treated. Underlying conditions contributing to causing the somatic dysfunction such as short leg should be addressed. Considerations for orthopedic or neurosurgical evaluation should be given when radiologic abnormalities such as scoliosis or spondylolisthesis are found on the screening examination.

\section{Discussion}

Ideally, all less than optimal health factors and habits would be brought up to a high standard before placement in jobs where low-back injury is a possibility. Obviously, this is not feasible because only certain factors can be tested and reliably retested without the possibility of being subjective or discriminatory. Much of the current literature cites poor general health as a significant cause of low-back morbidity, and improvement in overall health is an important factor in reducing the incidence of low-back pain. However, defining adequate health has remained difficult, and concrete testing for "adequate general health" is even more elusive.

In my view, the minimum level of fitness can be defined by the following criteria: (1) weight under the eightieth percentile for age; (2) passage of all the eight strength/flexibility tests described here; and (3) normal results of neurologic examination. These criteria can be tested easily and reproduced reliably between examiners without a great deal of subjectivity or possibility of discrimination.

It is up to the company to determine the following: what weight scale it will use to define the "normal" weight for age so that the eightieth percentile figure will be consistent for that company; what areas of the plant are classified as high risk for low-back injury; and whether to place an employee in that area on the basis of the examiner's findings.

The first two criteria, weight restrictions and minimum level of strength and flexibility, can be modified by the employee wishing placement in a high-risk area. The criteria are not difficult to meet, provided that the patient has a motivation to do so. An abnormal result on a neurologic examination should be evaluated thoroughly; it may indicate a problem that justifies excluding a patient from a particular work area, to protect the employee as well as the company. All other factors discussed should be screened and encouragement given in their improvement if needed. However, it is not recommended that they be used in the placement decision-making process.

If this protocol is adopted by the company, the physician should recommend that all employees in high-risk areas be retested every 6 to 12 months. Such retesting will encourage maintenance of the minimum health standards.

\section{Summary}

Some factors known to be associated with industrial low-back injuries have been discussed. A proposal for screening by the general practitioner of the employee prior to placement in an area of high risk for low-back injury has been offered. The osteopathic concept of structure/function interdependency has been considered and recommendations given for conditioning the employee to lessen the risk of industrial low-back pain.

\footnotetext{
1. Wiesel SW, Feffer HL, Rothman RH: Industrial Low Back Pain. Charlottesville, Va, The Michie Co, 1985.

2. Cailliet R: Low Back Pain Syndrome, ed 3. Philadelphia, FA Davis Co, 1981.

3. Spengler DM, Bigos SJ, Martin NA, et al: Back injuries in industry: A retrospective study. 1. Overview and cost analysis. Spine 1986;11:241245.

4. Morris A: Identifying workers at risk to back injury is not guesswork. Occupat Health Safety1985;55 (December):16-20.

5. White AH: Back School and Other Conservative Approaches to Pain. St Louis, CV Mosby Co, 1983.

6. Liang MG, Daltroy LH, Pallozzi LM: The patient's responsibility in therapy for LBP. J Musculoskel Med 1986;3 (July):43-46.

7. Kraus H. Backache Stress and Tension: Their Cause, Prevention and Treatment. New York, Simon \& Schuster, 1965, pp 8-11.

8. Norkin CC, White DJ: Measurement of joint motion: A guide to goniometry. Philadelphia, FA Davis Co, 1985, p 139.

9. Kappler RE: Role of psoas mechanism in low-back complaints. JAOA 1973;72:130-137.

10. Carron H: Acute low back pain. Emergency Decisions 1986;7/8:2639.

11. Mitchell FL: Structural pelvic function. American Academy of Osteopathy Yearbook, Newark, Ohio, 1965, pp 178-199.

12. Greeman PE: Innominate shear dysfunction in the sacroiliac syndrome. Manual Medicine, 1986;2:121-21.

13. Willman, MK: Radiographic technical aspects of the postural study. JAOA 1977;76:739-744.
}

From the Department of Osteopathic Manipulative Medicine, Kirksville College of Osteopathic Medicine, Kirksville, Mo.

Reprint requests to Dr Steele, KCOM, 800 W Jefferson St, Kirksville, MO 63501. 
त) STUART PHARMACEUTICALS

1987 ICl Americas Inc

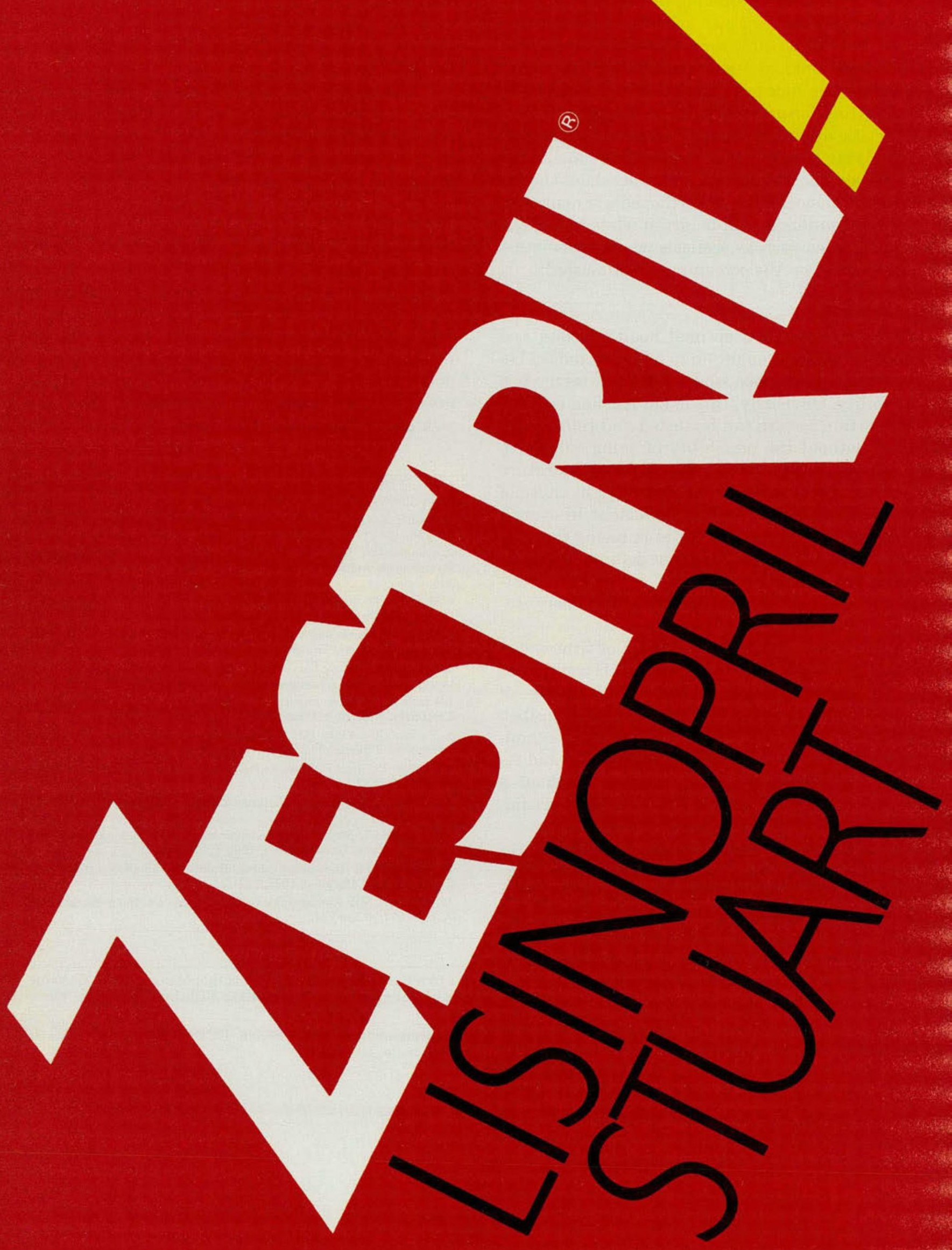

